\title{
Comparative Analysis of Various Soft Computing Techniques for Classification of Fruits
}

\author{
Harmandeep Singh \\ Research Scholar \\ I.K.Gujral Punjab Technical University \\ Jalandhar
}

\author{
Baljit Singh Khehra, PhD \\ Professor and Head \\ Dept. of CSE, BBSBEC \\ Fatehgarh Sahib
}

\begin{abstract}
Fruit classification and recognition techniques play a significant role in various fruit based computer vision systems. Many techniques have been proposed so far to classify and recognize fruit images. However, these techniques not provide efficient results whenever fruit images have poor visibility due to poor environmental conditions. The overall objective of this paper is to study and explore various techniques which have been designed so far to classify and recognize fruit images. Comparisons have been drawn between some well-known fruit classification and recognition techniques based upon certain features. It has been observed that soft computing based fruit classification and recognition techniques performs efficiently than existing techniques.
\end{abstract}

\section{Keywords}

Fruits; classifier; Soft computing; hybrid technique

\section{INTRODUCTION}

Fruits are the important part of our daily diet, that we eat, which offers tremendous benefits to human life. There are so many fruits that are available throughout the year and some fruits are seasonal. Indian economy is still very much depends on agriculture. India is ranked 3rd among the top producers of fruits in the world. So classification of fruits using soft computing based techniques is very much beneficial both for the producers and consumers. At present, computer vision is getting more involved in agriculture and production science. .Artificial Intelligence and many soft computing techniques are used for classifying fruits, to provide the better quality of the fruits to the consumers. Classification of Fruits is very important because it is very useful for improving fruit quality, recognizing different kind of fruits is a difficult task in a market, to categorize a fruit to determine its price manually is very difficult, counting manually the reaped fruits and determine its quality is a difficult task. Some major problem related to fruits producing, marketing and safe storing are: rising labour costs; shortage of skilled workers and go-down storing costs etc.

Automation in fruit industry, especially in agriculture comes into play to increase productivity and economic growth of the developing countries like india. Fruits and vegetables grading is an important task for producers, which affects the quality evaluation and export market but grading and sorting by human is a slow and labour intensive and also error prone and difficult task. So, there is a need of an automatic fruits grading system. In the past few years, researchers had developed numerous algorithms for sorting of fruits using computer vision based on colour, texture and morphological features to identify the diseases, maturity and class. Subsequently these features are used to train the soft computing based techniques to classify the fruits.
Soft computing vision systems provide considerable information about the nature and attribute of the fruits which reduces cost, guarantees the maintenance of quality information.

The classification model includes capturing, processing and analyzing images to facilitate the objective and assessment of visual quality characteristics. The techniques used in image analysis include image acquisition, image pre-processing and image interpretation, leading to quantification and classification of images and objects of interest within images. Classification techniques that are used for fruit classification are divided into the following phases:

a. Image Acquisition

b. Pre-processing

c. Feature Extraction

d. Classification

e. Evaluation

a. Image Acquisition: It is the creation of digital image, typically from a physical scene. The term assumed to imply or include processing, compression, storage, printing, and display of such image. Its name specifies definition. To get image from any source especially hardware based any source is called as image acquisition in the image processing.

b. Pre-processing: Pre-processing is a common name for operations with images at the lowest level of abstraction - both input and output are intensity images. The aim of pre-processing is an improvement of the image data that suppresses unwanted distortions or enhances some image features important for further processing.

c. Feature-Extraction: once pre-processing is completed, then features are collected in the feature extraction stage. Features, as described here, are real numbers obtained by applying some mathematical expression to image data, e.g. spatial domain pixel values or transformed spectral domain. Selection of features is the main task in recognition applications. Optimal feature subset selection increases the search rate and accuracy of the recognition system based on the performance of classifiers.

d. Classification: A classification problem deals with recognizing a given input with one of the distinct classifiers. There are several classifiers used to classify images. Object Classification is an important task within the field of computer vision. 
Image classification refers to the labelling of images into one of a number of predefined categories. Classification includes image sensors, image preprocessing, object detection, object segmentation, feature extraction and object classification.

e. Evaluation: Evaluation is the last phase of the classifier system. In this phase, proposed classifier is tested for testing patterns.

\section{COMPUTER VISION}

The computer vision strategies are used to recognise a fruit based on four main features which characterize the object intensity, colour, shape and shape. Computer vision system for quality evaluation of fruits have great acceptance in the food industry because they have increased demand for objectivity, consistency and efficiency in the evolution of quality. The automatic quality control of agricultural products has evolved along with the technology of image acquisition and processing speed of computers, many countries now use computer vision as an alternative to improve the quality control in the fruit industry. Most important work that implement computer vision technology is to improve the quality control in agriculture industry especially in fruits and vegetable products to:

- Identity stages of ripening with the help of acquired images

- Extracted the various shapes and size features related to the colour and texture

- Enhance and de-noise the acquired digital fruit images

- Segment the fruit images based on colour, shape, size and density

Following figure shows the computer vision system [2]

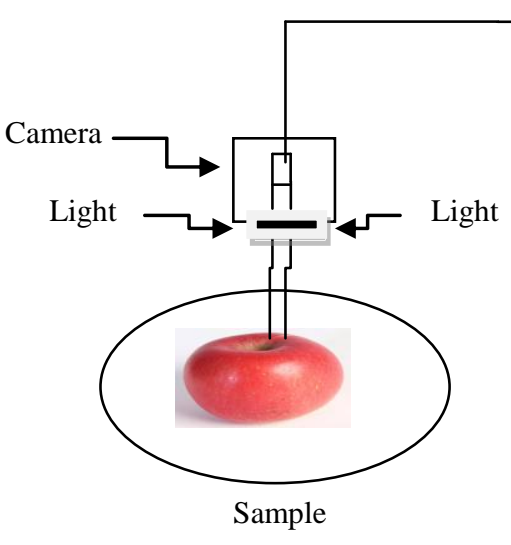

\section{HYBRID CLASSIFIER}

Hybrid classifiers means using the results of different classifiers are combined and then produced result is better as compared to the results produced by each classifier individually. Following are some important classifiers which are studied during literature survey and observed that they are used successfully in the classification of fruits in the future perspective for research purposes.

ANN: An Artificial Neural Network(ANN); BPN: Backpropagation Network:; SVM: Support Vector Machine; K-Means Clustering; KNN: K-nearst Neighbour; Genetic Algorithm; NFC: Neuro-fuzzy classifier; Fuzzy C-means; Ant Colony optimization; MDC: Minimum Distance Classifier ; MLC: Maximum Likelihood Classifier; RBF: Radial Basis function; PNN: Probabilistic Neural Network; Decision Tree; Moving K-means; N-cut Algorithm; Naive Bayes Classifier; Ranked c-means; Multi-class SVM; Bayesian Classifier

All the above mentioned classifiers are used by different researchers in the different classification purposes. In future some of the above classifiers should be use for classification of fruits based on the shape, colour and texture features and the classifiers which produce better accuracy should be combined to design a hybrid classifier model for classifying fruit images.

\subsection{Artificial Neural Network}

Artificial neural network have the tendency to get training from samples and also perform classification of unseen patterns. Clark [14], gives the simplest definition of an artificial neural network is provided as: "A computing system made up of a number of simple, highly interconnected processing elements, which process information by their dynamic state response to external inputs". Artificial neural networks are an attempt at modeling the information processing capabilities of nervous systems. An artificial neural network (ANN) is an information processing paradigm that is inspired by the way biological nervous systems, such as the brain, process information. The key element of this paradigm is the novel structure of the information processing system. It is composed of a large number of highly interconnected processing elements (neurons) working in unison to solve specific problems. ANNs, like people, learn by example. An ANN is configured for a specific application, such as pattern recognition or data classification through a learning process. Among all approaches, ANN is classified as having fastest speed and best accuracy for classification and recognition type of problems [13].

\subsection{Back Propagation Network (BPN):}

BPN is a method used in artificial neural networks to calculate the error contribution of each neuron after a batch of data (in image recognition, multiple images) is processed. This is used by an enveloping optimization algorithm to adjust the weight of each neuron, completing the learning process for that case.[40]

BPN is a generalization of the delta rule to multi-layered feedforward networks, made possible by using the chain rule to iteratively compute gradients for each layer. The backpropagation algorithm has been repeatedly rediscovered 
and is a special case of a more general technique called automatic differentiation in reverse accumulation mode. It is closely related to the Gauss-Newton algorithm, and is part of continuing research in neural BPN, is commonly used to train deep neural networks [1], a term used to describe neural networks with more than one hidden layer[40].

\subsection{Support Vector Machine(SVM)}

A SVM is a supervised machine learning algorithm used for data classification and estimating the relationships between variables (regression analysis). It's a "supervised" algorithm because there's an initial training phase involved where we feed the input data (algorithm) that has already been classified. After this initial (starting) training phase is completed, future data sets given to the algorithm can be classified with no or minimal human intervention. [39]

\subsection{Fuzzy C-Means}

Fuzzy clustering is a form of clustering in which each data point can belong to more than one cluster.Clustering or cluster analysis involves assigning data points to clusters such that items in the same cluster are as similar as possible, while items belonging to different clusters are as dissimilar as possible. Clusters are identified via similarity measures. These similarity measures include distance, connectivity, and intensity. Different similarity measures may be chosen based on the data or the application [41].

Fuzzy c-means (FCM) is a data clustering technique in which a dataset is grouped into $\mathrm{n}$ clusters with every datapoint in the dataset belonging to every cluster to a certain degree. For example, a certain datapoint that lies close to the center of a cluster will have a high degree of belonging or membership to that cluster and another datapoint that lies far away from the center of a cluster will have a low degree of belonging or membership to that cluster [42]

\subsection{Genetic Algorithms}

(GA) is a meta-heuristic inspired by the process of natural selection that belongs to the larger class of evolutionary algorithms (EA). Genetic algorithms are commonly used to generate solutions to optimization and search problems by relying on bio-inspired operators such as mutation, crossover and selection. [37]

The general form a of a genetic algorithm is the following [38]:

\section{Algorithm}

\section{Compute initial population;}

2. WHILE stopping condition not fulfilled

3. DO BEGIN Select individuals for reproduction;

4. Create offspring's by crossing individuals;

5. Eventually mutate some individuals;

6. Compute new generation

7. END

\section{LITERATURE SURVEY}

Giacomo et.al [1] presented a new approach that classifies fruit surface defects in colour and texture using RBPNN. The texture and gray features of defect area are extracted by computing a gray level co-occurrence matrix and then defect areas are classified by the applied RBPNN. They classify the fruits based on shape, colour and texture. In RBPNN classifier, they grouped the defects into classes. Class1: good quality fruits, class 2 :surface defect as bruises, class 3 : morphological defects, class4: slight color defects, class5: black mould.

G.S.gill et.al [2] proposed a good review of automatic fruit grading and classification using computer vision. They review the various classifiers that are used for classification of fruits based on computer vision and features such as color features, morphological features and texture features. They also discuss the application of computer vision system in fruit grading. In their paper they also discuss the brief summary of different fruits with their parameters and efficiency. They also define the most popular classification models such as (i) Fuzzy logic, ANN, ANFIS and SVM with their classification accuracy of different fruits.

VaniAshok and Dr. D.S.Vinod[3] proposed a fruit quality evaluation approach using probabilistic neural network based on the analysis of external features such as color, size, shape, texture and presence of damage. For quality evaluation they take sample of apple fruit images. Firstly they extract the features and then select the best features and evaluation the quality. They shown that the accuracy of classification by PNN is 86.52 .

Chandan k. Et.al[4] in their proposed system, developed an integrated system for grading of the defected fruits. The results shown that the performance is adequate to provide the basis for a viable commercial visual inspection system. In the propose

Abdulhamid H. et.al[9] proposed a method for automatic classification of date fruits based on computer vision and pattern recognition. The proposed method was implemented and empirically tested on different category of date fruit images. Finally they classify the extracted features with the help of nearest neighbour, linear discriminant analysis and neural network classifiers. Neural network shows the $98.6 \%$ accuracy during classification, LDA shows $96 \%$ and NN shown the $90 \%$. Accuracy during classification.

S.Janardhana et.al[10] reviews the recent development in the computer vision for agriculture and food industry. In their study they shows that the till date there is not such a machine vision or computer vision is developed for the automatic grading of foods in the food industry for quality inspection and gradation.

Gila d.et.al [11] proposed a hybrid technique of automatic fruits identification system as a combination of ANN (Artificial Neural Network), FD (Fourier descriptors) and SDA (Spatial domain analysis) for developing the automatic fruits identification and sorting. They capture fruit images using digital camera and segment the captured images into shape, color and dielectric based. They also filter the captured images to eliminate the unwanted interference and the noise from the acquired images. They recognize fruit color using FD and SDA techniques. Their results shown that the accuracy of classification is better using hybrid techniques as compared to the individual classifiers. From their proposed system and results it have been confirmed that the increasing the number of FD's greatly increase the accuracy of classification and decrease the classification time. FD's have the highest classification rate i.e., 99.1\%. From their results and classification it is observed that the hybrid techniques is more robust and classify the fruits better as compared to the classifiers used individually. 
Summary of research work discussed

\begin{tabular}{|c|c|c|c|c|c|}
\hline Title & Authors & Methods & Accuracy & Year & Benefits \\
\hline $\begin{array}{l}\text { Automatic Classification } \\
\text { of Fruits defects based on } \\
\text { co-occurence matrix and } \\
\text { neural networks }\end{array}$ & $\begin{array}{lr}\text { Giacomo } & \text { Capizzi, } \\
\text { Grazia Lo Sciuto, } \\
\text { Christian } & \text { Napoli, } \\
\text { Emiliano Tramontana, } \\
\text { Marcin Wozniak }\end{array}$ & $\begin{array}{lr}\text { Radial } & \text { Basis } \\
\text { Probabilistic } & \text { neural } \\
\text { network, } & \text { Grey } \\
\text { Level co-occurence } \\
\text { matrix }\end{array}$ & $94 \%$ & 2015 & $\begin{array}{ll}\text { 1. } & \text { Fast and } \\
\text { efficient } \\
\text { classification }\end{array}$ \\
\hline 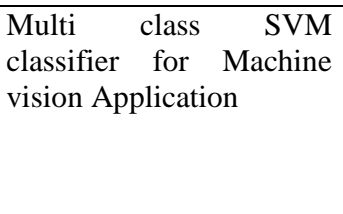 & $\begin{array}{l}\text { J.Suriya Prakash, K. } \\
\text { Annamalai Vignesh, } \\
\text { c.Ashok, R.Adithyan }\end{array}$ & $\begin{array}{l}\text { Multi Class SVM, } \\
\text { RBF, KNN, Naive } \\
\text { Bayes Classifiers }\end{array}$ & $\begin{array}{l}\text { MCSVM }=92 \% \\
\text { KNN }=90 \% \\
\text { Naive bayes }=84 \\
5\end{array}$ & 2012 & $\begin{array}{l}\text { 1. Efficient data } \\
\text { classification } \\
\text { 2. MCSVM is superior } \\
\text { as compared with } \\
\text { KNN and RBF }\end{array}$ \\
\hline $\begin{array}{l}\text { Fitting a pineapple model } \\
\text { for automatic maturity } \\
\text { grading }\end{array}$ & $\begin{array}{l}\text { Watcharin } \\
\text { Kaewapichal, Pakorn } \\
\text { Kaewtrakulpong, Asa } \\
\text { Prateepasen, Kittiya } \\
\text { Khongkraphan }\end{array}$ & $\begin{array}{l}\text { Modofied } \\
\text { algorithm }\end{array}$ & $90 \%$ & 2007 & $\begin{array}{lr}\text { I.Increased } & \text { fitting } \\
\text { accuracy } & \text { and } \\
\text { efficiency } & \end{array}$ \\
\hline $\begin{array}{l}\text { Classification of citrus } \\
\text { fruit using image } \\
\text { processing-GLCM } \\
\text { parameters }\end{array}$ & $\begin{array}{l}\text { Chandan Kumar, } \\
\text { Siddharth Chauhan, R. } \\
\text { Narmada Alla, Harika } \\
\text { Mounica Gurram }\end{array}$ & $\begin{array}{l}\text { Grey level co- } \\
\text { occurence matrix } \\
\text { method }\end{array}$ & $91 \%$ & 2015 & $\begin{array}{l}\text { 1.Enhanced the } \\
\text { contrast of the fruit } \\
\text { images }\end{array}$ \\
\hline $\begin{array}{l}\begin{array}{l}\text { Detection } \\
\text { classification of }\end{array} \text { apple } \\
\text { fruit diseases using } \\
\text { complete local binary } \\
\text { patterns }\end{array}$ & $\begin{array}{l}\text { Shiv Ram Dubey, } \\
\text { anand singh lal }\end{array}$ & $\begin{array}{l}\text { K-means clustering, } \\
\text { multi class SVM }\end{array}$ & $93 \%$ & 2012 & $\begin{array}{l}\text { 1.significantly support } \\
\text { accurate detection and } \\
\text { automatic } \\
\text { classification of fruit } \\
\text { diseases. }\end{array}$ \\
\hline $\begin{array}{l}\text { Automatic Fruit Grading } \\
\text { and classification using } \\
\text { computer vision: A } \\
\text { Review }\end{array}$ & $\begin{array}{l}\text { Seema, A.Kumar, } \\
\text { G.S.Gill }\end{array}$ & $\begin{array}{l}\text { Fuzzy Logic, ANN, } \\
\text { SVM, ANFIS }\end{array}$ & $91 \%$ & 2015 & $\begin{array}{l}\text { Performance improved } \\
\text { as compared to } \\
\text { previous classification } \\
\text { methods }\end{array}$ \\
\hline $\begin{array}{l}\text { Automatic Quality } \\
\text { Evaluation of Fruits using } \\
\text { Probabilistic Neural } \\
\text { Network approach }\end{array}$ & $\begin{array}{l}\text { Vani Ashok, } \\
\text { Dr. D.S.Vinod }\end{array}$ & PNN Classifier, & $88.33 \%$ & 2014 & $\begin{array}{l}\text { 1.the classification } \\
\text { performance improves } \\
\text { with increase in the } \\
\text { number of features }\end{array}$ \\
\hline $\begin{array}{ll}\text { Automatic } & \text { Fruits } \\
\text { Identification } & \text { using } \\
\text { Hybrid Technique } & \end{array}$ & $\begin{array}{l}\text { A.M. Aibinu, M.J.E. } \\
\text { Salami, A.A.Shafie, } \\
\text { N.Hazali, N.Termidzi }\end{array}$ & ANN, FD, SDA & $99.1 \%$ & 2011 & $\begin{array}{lr}\text { 1.Hybrid } & \text { classifiers } \\
\text { performs } & \text { better } \\
\text { classification } & \end{array}$ \\
\hline
\end{tabular}

\section{CONCLUSION}

The paper has presented a comprehensive review on various classification and recognition techniques which have been designed for fruit images. These techniques have been compared with each other based upon certain features. It has been observed that soft computing based techniques outperforms existing techniques. Also, existing techniques perform poorly for weather degraded fruit images. Therefore, in near future various techniques will be explored and implemented as a pre-processing tool to improve the accuracy rate of existing fruit classification and recognition techniques.

\section{REFERENCES}

[1] Giacomo C. Et.al, (2015). Automatic Classification of Friut Defects based on Co-occurence Matrix and Neural Networks. Proceedings of the Federated Conference on Computer Science and Information systems pp.861-867

[2] Seema et.al, (2015). Automatic Fruits Grading and Classification system using Computer Vision: A Review. Second International Conference on Advances in Computing and Communication Engineering pp.598-603.
[3] VaniAshok et.al, (2014). Automatic Quality Evaluation of Fruits using Probabilistic Neural Network Approach. International Conference on Contemporary Computing and Informatics(IC3I) pp.308-311.

[4] Chandan K. Et.al, (2015).

[5] Shiv R. Et.al,(2012). Detection and classification of Apple Fruit Diseases using Complete Local Binary Patterns. Third International Conference on Computer and Communication Technology pp.346-351.

[6] Suriya J. Et.al, (2012). Multi Class Support Vector Machine Classifier for Machine Vision Application. International Conference on Communications and Signal Processing (ICCSP) pp.0001-0005.

[7] Watcharin K. Et.al, (2007). Fitting a Pineapple Model for Automatic Maturity Grading. IEEE ICIP pp.257-260.

[8] Yasuo S. Et.al, (2003). Eggplant classification using Artificial Neural Network. pp.1013-1018.

[9] AbdulHamid. H et.al, (2012). Image-Based Date Fruit 
Classification. IV International Congress on Ultra Modern Telecommunications and control systems. pp.357-361

[10] S.Janardhana et.al, (2013) Computer Aided Inspection system For Food Products using Machine Vision: a Review. International conference on current Trends in Engineering and Technology, ICCTET,2013 pp.29-33.

[11] A.M Aibinu et.al, (2011) Automatic Fruits Identification system Using Hybrid Technique Sixth IEEE International Symposium on Electronic Design, Test and Application. pp.217-221.

[12] Fabio A.F. et.al, (2012) Automatic Classifier Fusion for Produce Recognition. XXV SIBGRAPI Conference on Graphics, Patterns and Images. pp.252-259.

[13] Lin, H. et.al, 2008. Machine Recognition for BroadLeaved Trees Based on Synthetic Features of Leaves Using Probabilistic Neural Network. Proceedings of IEEE International Conference on Computer Science and Software Engineering, 12-14 Dec. Wuhan, Hubei, China, pp.871-887.

[14] Philips, R. D., et al. 2014. An SMP Soft classification algorithm for remote sensing. Computers \& Geosciences. vol. 68 , pp.73-80.

[15] Liu, C. et.al, 2001. A Shape and Texture Based Enhanced Fisher Classifier for Face Recognition. IEEE Transactions on Image Processing. vol. 10, no. 4, pp.598-608.

[16] Kishitij, F. et.al, 2014. Detection and classification of plant diseases. International Journal of Engineering Research and General Science. vol.2, no.6, pp.868-874.

[17] Owirka, G.J. et.al, Template-Based SAR ATR Performance Using Different Image Enhancement Techniques. MIT Lincoln Laboratory, pp.1-13.

[18] Das, R. et.al, 2012. Image Segmentation Techniques. IJECT vol. 3, no.1, pp.66-70

[19] Trier, O. et.al, 1996. Feature Extraction Methods for Character Recognition: A survey. Pattern Recognition. Vol.26, no.4, pp.641-662.

[20] Harvey, N.R. et.al, 2002. Comparison of GENIE and Conventional Supervised Classifiers for Multispectral Image Feature Extraction. IEEE Transactions on Geoscience and Remote Sensing. FEBRUARY 2002. vol. 40 , no. 2

[21] Khehra, B.S. and Singh. A.P. 2011. Digital Mammogram Segmentation using Non-Shannon Measures of Entropy. Proceedings of the World Congress on Engineering. WCE 2011, London, U.K. Vol.2

[22] Anke, M.B. 2004. Pattern recognition for medical imaging. Elsevier Academic Press, UK, pp.14-18.

[23] Li, H. et.al, 2001. Computerized radiographic mass detection-part-II: lesion site selection by morphological enhancement and contextual segmentation. IEEE
Transactions on medical imaging. vol.20, no. 4, pp.302313.

[24] Wei, L. et.al, 2005. A study of several machine- learning methods for classification of malignant and benign clustered microcalcifications. IEEE transactions on Medical Imaging. vol. 24, no.3, pp.371-380,

[25] Cheng, H.D, 2006. Approaches for automated detection and classification of masses in mammograms. Pattern Recognition. vol.39, pp.646-668.

[26] Ross, p. et.al, 2002. Learning a Procedure That Can Solve Hard Bin-Packing Problems: A New GA-Based Approach to Hyper-heuristics. European Journal of Operational Research. vol.137, no.1, pp. 1295-1306.

[27] Kim, M.J. et.al, 2006. An evolutionary approach to the combination of multiple classifiers to predict a stock price index. Expert Systems with Applications. vol.31, no.2, pp.241-247.

[28] Ho, S.Y. et.al, 2004. Design of accurate classifiers with a compact fuzzy-rule base using an evolutionary scatter partition of feature space. IEEE Transactions on Systems, Man, and Cybernetics, Part B: Cybernetics focuses on cybernetics, including communication and control across humans, machines and organizations at the structural or neural level. vol.34, no.2, pp.1031-1044.

[29] Burke, E.K.et.al, 2010. A Classification of Hyperheuristic Approaches. Handbook of Meta-heuristics International Series in Operations Research \& Management Science. vol.146, pp .449-468.

[30] Villiammal, N. et.al, 2012. Efficient feature fusion, selection \& classification technique for plant leaf image retrieval system. Proceedings of ACM 2nd International Conference on Computational Science, Engineering and Information Technology, 26-28 Oct. Coimbatore Tamil Nadu, India, pp.132-137.

[31] Gill,J. et.al, 2014. A Review of Automatic Fruit Classification Using Soft Computing Techniques. IJCSEE (online). Vol.2, no.2, pp.99-105.

[32] Cho, S. B. 1997. Neural Network classifiers for Recognizing Totally Unconstrained Handwritten Numerals. IEEE Tranactioms on Neural Network. vol.8, no.1, pp.209-310

[33] https://en.wikipedia.org/wiki/Genetic_algorithm

[34] D.M. Mukhopadhyay, M.O. Balitanas, A. Farkhod, S.H. Jeon, and D. Bhattacharyya, Genetic Algorithm: A Tutorial Review, International Journal of of Grid and Distributed Computing, Vol.2, No.3, 2009, pp. 25-32

[35] http://subtlearray.tumblr.com/post/42779370765

[36] https://en.wikipedia.org/wiki/Backpropagation

[37] https://en.wikipedia.org/wiki/Fuzzy_clustering 